Original Contribution

\title{
INTRA- AND INTER-CULTIVAR GENETIC VARIABILITY IN BULGARIAN TOBACCO
}

\author{
B. Bojinov ${ }^{1}$, R. Bozhinova ${ }^{2}$ \\ ${ }^{1}$ Department of Genetics and Plant Breeding, Agricultural University of Plovdiv, Plovdiv, Bulgaria \\ ${ }^{2}$ Tobacco and Tobacco Products Institute, Plovdiv, Bulgaria
}

\begin{abstract}
PURPOSE: The genus Nicotiana is a well-defined group of species of which tobacco (Nicotiana tabacum L.) is the most important crop plant and plays a significant role in the economies of many countries. Recent advances in molecular genetics of the crop allowed the identification of many important genes and their location on chromosomes. However, the genetic analysis of this inbreeding, highly homozygous plant that serves as a model in many other studies is still very much work in progress.

METHODS: A large proportion of the tobacco genome is represented by highly repeated DNA sequences, which makes molecular markers, based on them, an obvious first choice in diversity studies. Here we present an assessment with Inter-Simple Sequence Repeat (ISSR) markers of the intra- and inter-cultivar variability in a set of Bulgarian tobacco varieties.

RESULTS: The screening of the 4 primers revealed a varying number of bands generated by different primers. The total number of bands varied between 4 and 13. No correlation between the total number of bands and the number of polymorphic bands was observed. Testing with ISSR primers revealed that plants with different DNA profiles can be found in varieties used. The highly similar profiles when E7 primer was used in two of the tested varieties from different production types indicate that the DNA fingerprinting technique may present a particular challenge in this crop.

CONCLUSIONS: The present study demonstrates the power of the ISSR technique as a tool for diversity identification in tobacco.
\end{abstract}

Key words: Nicotiana tabacum, ISSR markers, DNA polymorphisms, DNA fingerprinting

\section{INTRODUCTION}

The genus Nicotiana [1] is a well-defined group of species of which tobacco (Nicotiana tabacum L.) is the most important crop plant and plays a significant role in the economies of many countries [2]. Furthermore, tobacco is one of the most important model systems in plant biotechnology. Nicotiana species are often used in model experiments to demonstrate the synthesis of secondary metabolites, principles of disease resistance, and explore other basic questions of plant physiology.

Recent advances in molecular genetics of the crop allowed the identification of many

\footnotetext{
"Correspondence to: Bojin Bojinov; 12Mendeleev str., 4000 Plovdiv, Bulgaria; telephone number 00359898229 328; fax number - 0035932654 322; email address - bojinov@au-plovdiv.bg
}

important genes and their location on chromosomes [3]. However, the genetic analysis of this inbreeding, highly homozygous plant that serves as a model in many other studies is still very much work in progress. Few molecular marker analyses have been performed in allotetraploid tobacco (Nicotiana tabacum) such as the analysis of genetic relationships in wild and cultivated tobacco material [4-6], tagging of individual disease resistance genes with molecular markers such as RAPDs and AFLPs [7, 8], or expression of multiple transgenes, carrying such resistance [9]. Reasons for this are most likely that Nicotiana tabacum is a tetraploid species. The plant is considered to be an allopolyploid interspecific hybrid between Nicotiana sylvestris $(\mathrm{n}=12)$ and Nicotiana tomentosiformis $(\mathrm{n}=12)$ and has $\mathrm{n}=24$ chromosomes $[10,11]$. A major obstacle in the development of a detailed genetic map of the 
tobacco genome is that the size of the tobacco genome is at the high end in the genus Solanaceae [12] with approximately 4,500 Mbp. A large proportion of the tobacco genome is represented by highly repeated DNA sequences including rDNA [13, 14], active transposons [15] and retrotransposons [16].

Through the efforts of the Tobacco Genome Initiative

(TGI,

http://www.tobaccogenome.org) efforts have been undertaken regarding a systematic characterization of the tobacco genome [17]. Considerable progress has been made since the initiation of TGI but this project lacked until recently a genetic component comprising the construction of a high-resolution genetic map that could provide a framework for anchoring the sequencing information to the tobacco chromosomes [18-21].

The above developments potentially could bring the knowledge and the tools for the application of molecular marker-assisted selection (MAS) in tobacco breeding programs. However, at present, the practical use of MAS is restricted not only by the novelty of the skills to be acquired by the majority of tobacco breeders but by the current high price of development and deployment of such markers as well. On the other hand, molecular markers could much quicker contribute to another field of significant interest to breeders - the need to uniquely identify ("fingerprint") varieties and breeding lines, preferably by using procedures that are based on an objective scoring of differences between the genotypes. Once obtained, such fingerprints could have multiple uses: reducing the redundancy in breeding collections, reducing the number of reference varieties in breeder's trials, developing variety "ID cards" for plant breeders' rights (PBR) protection, diversity studies, etc. These potential benefits have long been recognized and inspired several reports on the use of different fingerprinting techniques in other crops [22-25]. However most of these studies are based on comparisons of several genotypes from different wild species to relatively small groups of genotypes from cultivated ones, therefore stressing mainly on the opportunities for using DNA fingerprinting in diversity and phylogeny studies. As a first step towards the practical application of this technique in tobacco here we present a study on the intra- and intercultivar variability in a set of Bulgarian tobacco varieties.

\section{MATERIALS AND METHODS Plant materials}

Four commercial varieties from the two most widely grown tobacco types in Bulgaria (oriental and Burley) were included in this study. Eight plants from each - three oriental cultivars (Plovdiv 7, Nevrokop 1146 and Krumovgrad 90) and one of Burley (Burley 1317) that are widely grown in our country were used to assess the existing intra-cultivar diversity (Figure 1).
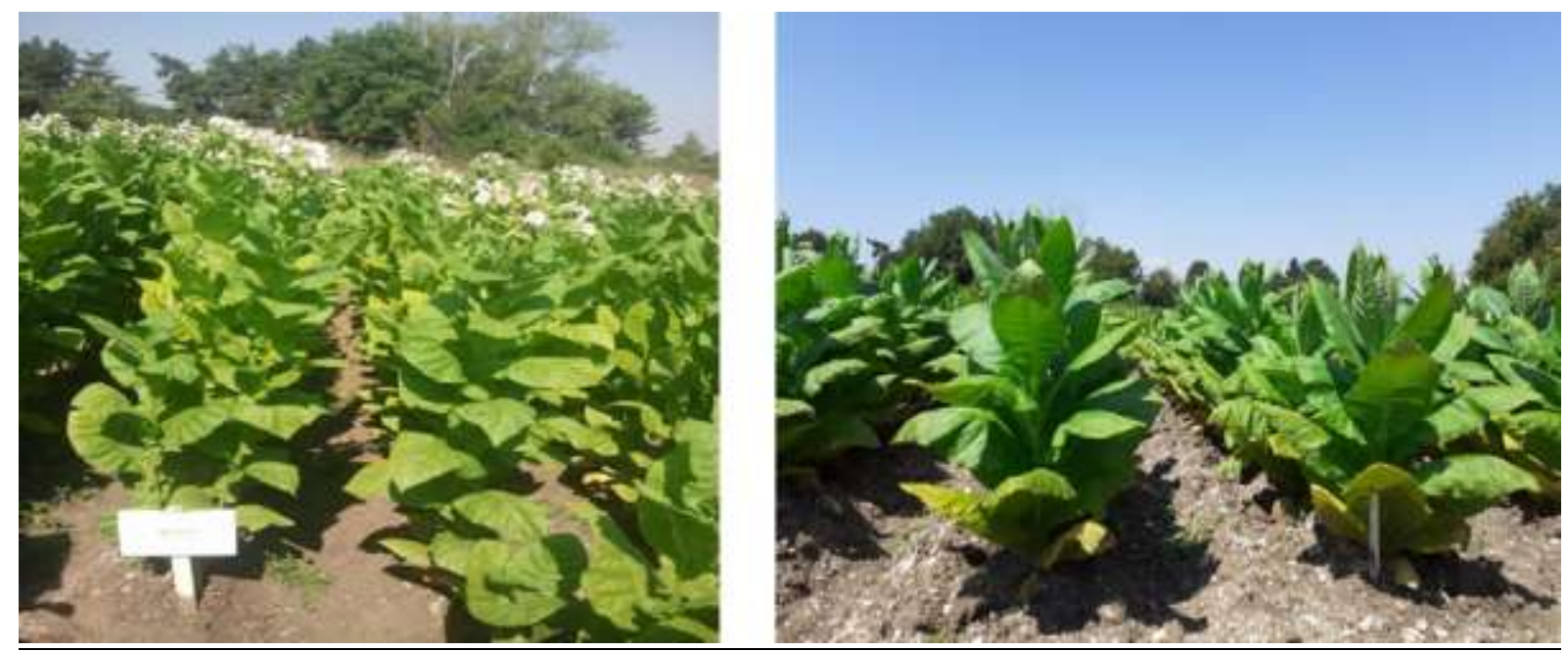

Figure 1. Plants from oriental tobacco Krumovgrad 90 (left) and Burley tobacco Burley 1317 (right) cultivars used in the present study. 


\section{DNA extraction}

DNA was extracted from the last fully expanded leaf of greenhouse-grown plants. Approximately $200 \mu \mathrm{g}$ leaf samples were frozen in liquid nitrogen and ground in a mortar. DNA from each accession was extracted using a standard DNA extraction kit (Nucleon PhytoPure, Amersham Biosciences UK Ltd., Buckinghamshire, HP7 9NA England) with minor modifications of the manufacturer protocol.

\section{ISSR analysis}

A total of 4 primers were tested with their sequences presented in Table 1. The presence or absence of each ISSR fragment was scored as a binary unit character $(1=$ present, $0=$ absent).

PCR reactions were performed in $25 \mathrm{ul}$ volume with the following cycling regime: denaturing at $94^{\circ} \mathrm{C}$ for $3 \mathrm{~min}, 40$ cycles of $94^{\circ} \mathrm{C}-1 \mathrm{~min}$, AT $-45 \mathrm{sec}, 72^{\circ} \mathrm{C}-45 \mathrm{sec}$, followed by final extension of $72^{\circ} \mathrm{C}-4 \mathrm{~min}$, where AT is the annealing temperature for each primer calculated according to [26].

PCR products were analyzed through separation in $2 \%$ agarose gels and staining with ethidium bromide.

Table 1. Primers tested for variety identification.

\begin{tabular}{|l|l|l|}
\hline Primer & DNA sequence & Length (bp) \\
\hline ISSR 1 & $(\mathrm{CA})_{8} \mathrm{AA}+\mathrm{GG}$ & $\mathbf{2 0}$ \\
\hline ISSR 2 & $(\mathrm{CA})_{8} \mathrm{AA}+\mathrm{GC}+\mathrm{T}$ & $\mathbf{2 1}$ \\
\hline ISSR 6 & $(\mathrm{AC})_{8} \mathrm{C}+\mathrm{TG}$ & $\mathbf{1 9}$ \\
\hline ISSR 7 & $(\mathrm{AG})_{8} \mathrm{C}+\mathrm{TG}$ & $\mathbf{1 9}$ \\
\hline
\end{tabular}

\section{RESULTS}

The screening of the 4 ISSR primers revealed a varying number of bands generated by different primers. The total number of bands varied between 13 for the ISSR-E7 and 4 for the ISSR-E2 primer. Similarly, the number of polymorphic bands was variable. No correlation between the total number of bands and the number of polymorphic bands was observed.

One notable observation during this initial testing was the presence of polymorphisms between the two plants of the Nevrokop 1146 variety (Figure 2). The two plants each had one band present, which was not present in the other plant of the same cultivar.

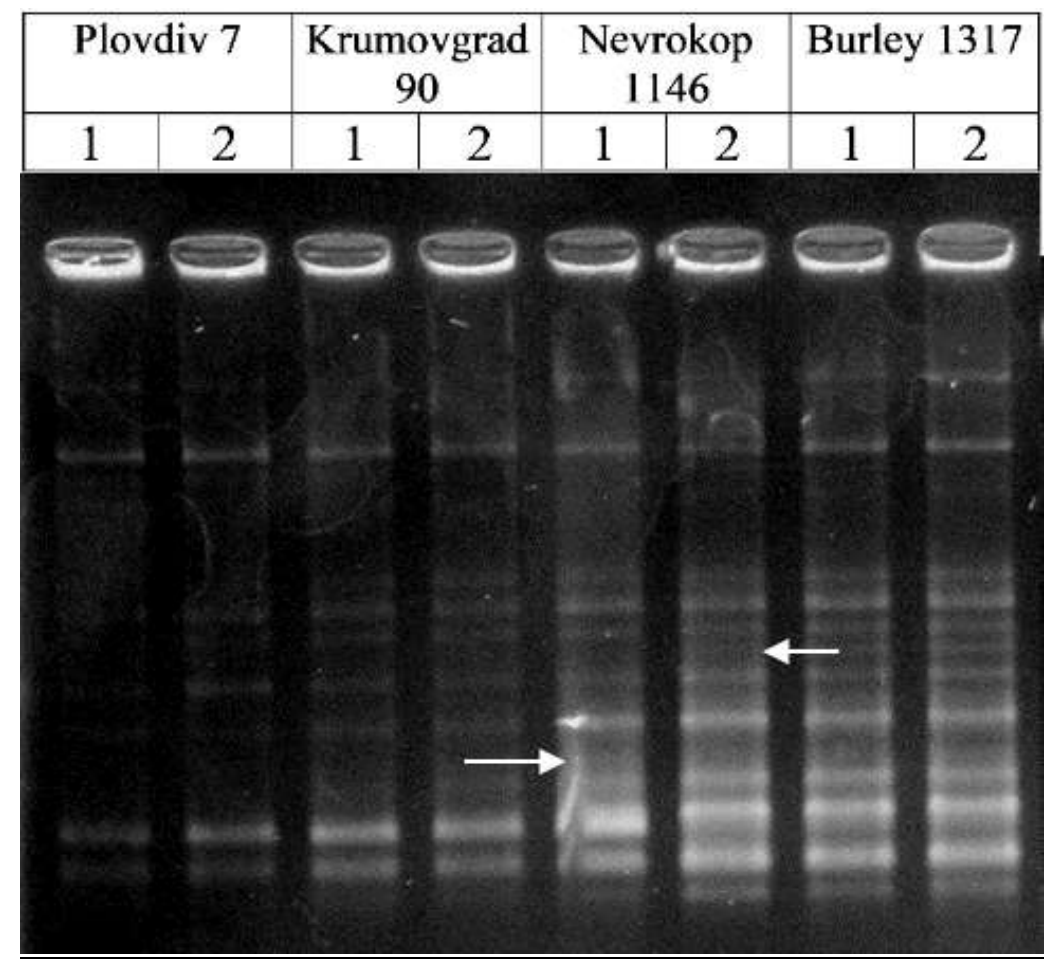

Figure 2. Observed polymorphisms in the initial testing with ISSR-E7 primer. Each cultivar is represented by two plants. Arrows point to the polymorphic bands between two plants of Nevrokop 1146 variety. 
Further testing revealed that plants with different DNA profiles can be found in other varieties, too (Figure 3). What is even more interesting is that the use of primer E6 revealed polymorphisms within the Burley 1317 variety (indicating that two of the plants differ from the rest) while producing essentially identical profiles for the two varieties - Burley 1317 and Nevrokop 1146 . The highly similar profiles for these two varieties from different production types obtained when E7 primer was used (Figure 2) indicate that the DNA fingerprinting technique may present a particular challenge in this crop.

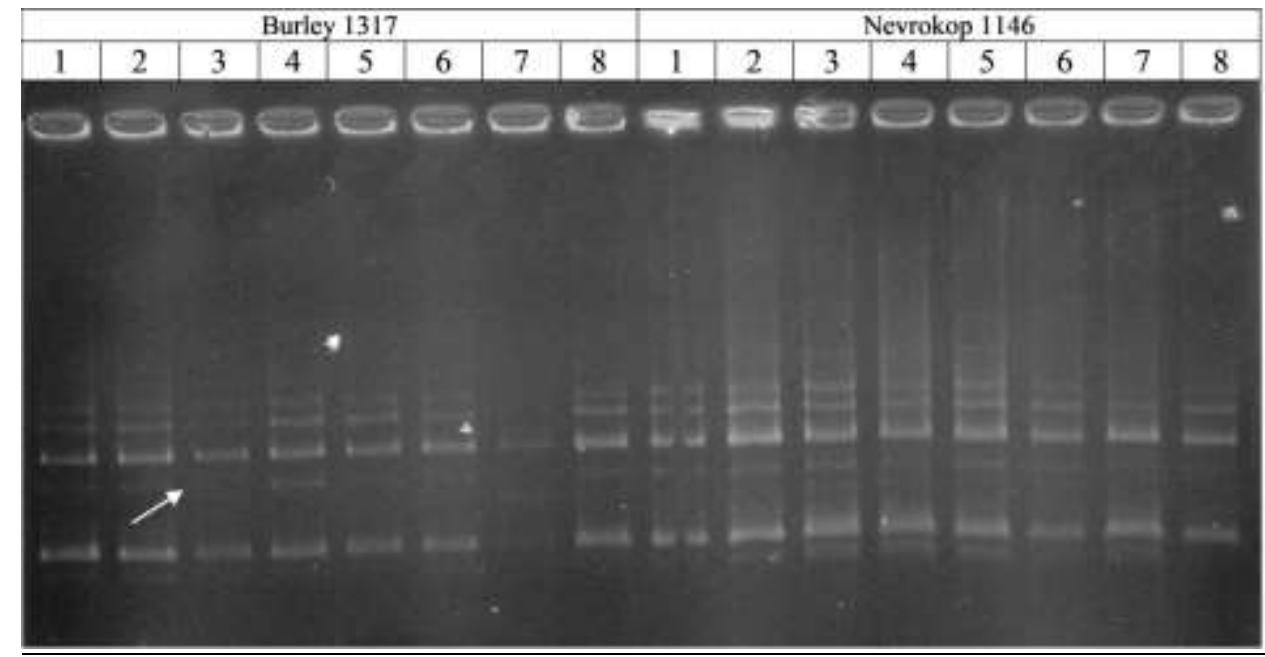

Figure 3. Observed polymorphisms in the testing of cultivars Burley 1317 and Nevrokop 1146 with ISSR-E6 primer. Each cultivar is represented by eight plants. Arrow points to the polymorphic band between plants of Burley 1317 variety.

\section{DISCUSSION}

When developing a DNA fingerprinting technique for variety detection one consideration that has to be taken into account is the determination of within-variety polymorphism levels like the one observed in the present study (Figure 2). This is important not only for the robustness of the variety identification but for the determination of the significance of the differences in terms of distinctness (assigning studied genotype to existing variety or giving it a status of a new one). Extended experimentation should be performed by each variety testing authority before fully adopting DNA fingerprinting. Assuming that (for practical reasons) not more than 4 primer couples will be used in such distinctness and uniformity testing this will allow for keeping low the costs of determination of within-variety polymorphism in each authority. The current testing system, which is based on the assessment of a range of standardized morphological characters, is not only expensive and time-consuming but often relies on subjective decisions of the personnel involved in the evaluation. Several types of molecular markers provide what could be an alternative to this situation. However, the reliability and reproducibility of microsatellite markers (SSRs) and amplified fragment length polymorphisms (AFLPs) make these two marker systems preferred when DNA fingerprinting is attempted in many different plant species [27-29]. Our previous comparative study on the potential of SSRs and AFLPs for cotton fingerprinting [30] has outlined the respective advantages of these two types of markers depending on the size of the population, its diversity, and the availability of required equipment and permissions. As discussed in that report, at present AFLPs would be the technique of choice when large populations have to be fingerprinted.

We consider previously available data on the effect of primer sequence on the number of generated polymorphic bands irrelevant in the particular case of selecting primers for fingerprinting, as the highly skewed markers, which contribute significantly to increasing the polymorphic information content (PIC) of primer couples, would be heavily selected against. Detection of such a combination of primers would allow for a significant reduction of the labour and consumables needed when other sets will have to be fingerprinted. Knowing the danger of extrapolating this kind of data to genotypes that were not included in 
the present study, the above suggestion should be taken with precaution.

In the future, the analysis of additional tobacco lines using a set of high-quality microsatellite markers should provide a very detailed picture of the genetic relatedness of tobacco lines within and between individual groups of germplasm. Furthermore, these data show that a limited set of microsatellite markers should be sufficient for variety identification and grouping of tobacco lines and varieties through the use of microsatellite marker databases.

\section{CONCLUSIONS}

The present study demonstrates the power of the ISSR technique as a tool for diversity identification in tobacco. Being long proposed, but not fully explored before, here it was studied through evaluating the capacity of several primers to produce polymorphisms in the two main cultivated tobacco types. However, further fine-tuning of the technique will be needed before it could be implemented to the full of its capacity - in DUS testing, PBR protection, sorting out of breeders' collections and genebanks from redundant entries, developing genotype "ID cards", etc.

\section{REFERENCES}

1. Goodspeed, T.H., The genus Nicotiana; origins, relationships, and evolution of its species in the light of their distribution, morphology, and cytogenetics. Chronica botanica,v. 16, no. 1/6., Waltham, Mass.: Chronica Botanica Co. 536, USA, 1954.

2. Davis, D.L. and M.T. Nielsen, Tobacco: production, chemistry and technology. Wiley-Blackwell, USA, 1999.

3. Sierro, N., et al., The tobacco genome sequence and its comparison with those of tomato and potato. Nature Comm, 5(1): p. 3833, 2014..

4. Bogani, P., et al., A Physiological and Molecular Analysis of the Genus Nicotiana. Mol Phyl Evol. 7(1): p. 6270, 1997.

5. Ren, N. and M.P. Timko, AFLP analysis of genetic polymorphism and evolutionary relationships among cultivated and wild Nicotiana species. Genome. 44(4): p. 559-571, 2001.
6. Rossi, L., et al., Potential of molecular marker analysis for variety identification in processed tobacco. Plant Var Seed. 14(2): p. 89-101, 2001.

7. Julio, E., J. Verrier, and F.D. De Borne, Development of SCAR markers linked to three disease resistances based on AFLP within Nicotiana tabacum L. Theor Appl Genet. 112(2): p. 335-346, 2006.

8. Nishi, T., et al., Identification of DNA markers of tobacco linked to bacterial wilt resistance. Theor Appl Genet. 106(4): p. 765-770, 2003.

9. Christova, P., R. Batchvarova, and N. Christov, Expression of four transgenes in one tobacco line. Bulg $J$ Ag Sci. 22(3): p. 437-441, 2016.

10. Kenton, A., et al., Characterization of the Nicotiana tabacum L. genome by molecular cytogenetics. Mol Gen Genet MGG. 240(2): p. 159-169, 1993.

11. Lim, K., et al., Genome evolution in allotetraploid Nicotiana. Biol J Linn Soc. 82(4): p. 599-606, 2004.

12. Arumuganathan, K. and E. Earle, Nuclear DNA content of some important plant species. Plant $\mathrm{Mol}$ Biol Rep. 9(3): p. 208-218, 1991.

13. Borisjuk, N.V., et al., Structural analysis of rDNA in the genus Nicotiana. Plant Mol Biol. 35(5): p. 655-660, 1997.

14. Volkov, R.A., et al., Elimination and rearrangement of parental rDNA in the allotetraploid Nicotiana tabacum. Mol Biol Evol. 16(3): p. 311-320, 1999.

15. Casacuberta, J.M., S. Vernhettes, and M.-A. Grandbastien, Sequence variability within the tobacco retrotransposon Tnt1 population. The EMBO journal. 14(11): p. 2670-2678, 1995.

16. Gregor, W., et al., A distinct endogenous pararetrovirus family in Nicotiana tomentosiformis, a diploid progenitor of polyploid tobacco. Plant Physiol. 134(3): p. 1191-1199, 2004.

17. Gadani, F., et al. Large scale genome sequencing and analysis of Nicotiana 
tabacum: the tobacco genome initiative. in 5èmes Journées Scientifiques du Tabac de Bergerac5th Bergerac Tobacco Scientific Meeting, Bergerac, France. 2003.

18. Bindler, G., et al., A high density genetic map of tobacco (Nicotiana tabacum L.) obtained from large scale microsatellite marker development. Theor Appl Genet. 123(2): p. 219-230, 2011.

19. Bindler, G., et al., A microsatellite marker based linkage map of tobacco. Theor Appl Genet. 114(2): p. 341-349, 2007.

20. Darvishzadeh, R., et al., Study on genetic diversity among Iranian water pipe's tobacco (Nicotiana $\mathrm{s} \quad \mathrm{pp}$.) varieties by using simple sequence repeat markers. Bulg J Ag Sci. 19(3): p. 557-562, 2013.

21. Tong, Z., et al., Large-scale development of SSR markers in tobacco and construction of a linkage map in flue-cured tobacco. Breed Sci. 66(3): p. 381-390, 2016.

22. Amer, A., et al., Applicability of Different Molecular Markers Techniques for Genetic Distinguish Between Two Genera <I $>$ Cressa $</$ I $>$ Linn. and $<$ I $>$ Cuscuta $</$ I $>$ Yunck. Family Convolvulaceae. Pak J Biol Sci. 21(4): p. 179-186, 2018.

23. El-Bakatoushi, R. and D.G.A. Ahmed, Evaluation of genetic diversity in wild populations of Peganum harmala L., a medicinal plant. $J$ Genet Eng Biotechnol. 16(1): p. 143-151, 2018.
24. Patel, P., et al., Assessment of genetic diversity in Colletotrichum falcatum Went accessions based on RAPD and ISSR markers. $J$ Genet Eng Biotechnol. 16(1): p. 153-159, 2018.

25. Sanchez-Velazquez, J.U. and A. Ramos-Diaz, ISSR diversity in Jatropha curcas germplasm and offspring of selected parentals. Data Brief. 20: p. 761-766, 2018.

26. Kochieva, E.Z., et al., [Genetic diversity and phylogenetic relationships of the genus Lycopersicon (Tourn.) Mill. as revealed by inter-simple sequence repeat (ISSR) analysis]. Genetika. 38(8): p. 1133-42, 2002.

27. Chen YiHua, et al., Rice seed identification by computerized AFLPDNA fingerprinting. CRRN, Chin Rice Res Newslet. 8(1): p. 4-5, 2000.

28. Faccioli, P., et al., Amplified fragment length polymorphism (AFLP) markers for barley malt fingerprinting. $\mathrm{J} \mathrm{Cer}$ Sci. 29(3): p. 257-260, 1999.

29. Garg, M., et al., Estimates of genetic similarities and DNA fingerprinting of wheats (Triticum species) and triticale cultivars using molecular markers. Ind J Ag Sci. 71(7): p. 438-443, 2001.

30. Bojinov, B. and J. Lacape, Molecular markers for DNA fingerprinting in cotton, in World Cotton Res. Conf. 3: Cotton production for the new millennium. p. 9-13, ARC-IIC, FAO. Rustenburg: Cape Town, South Africa, 2003. 